\title{
Balance de los Objetivos de Desarrollo del Milenio y su impacto en el derecho a la educación
}

\author{
ISABEL CARRILLO FLORES \\ Universidad de Vic-Universidad Central de Cataluña - España
}

Recibido el 28-11-2015; primera evaluación el 23-03-2016; segunda evaluación el 13-04-2016; tercera evaluación 04-05-2016; aceptado el 14-05-2016

\section{Resumen}

En este artículo se analiza la educación como derecho humano fundamental. El legado del siglo veinte muestra avances pero las crisis económicas y las políticas de ajuste adoptadas han agravado las desigualdades educativas. En este contexto los Objetivos de Desarrollo del Milenio proyectados para el 2015 han quedado limitados. Primero se significa la educación como derecho humano. En segundo lugar se hace balance de los objetivos y se exponen sus impactos en la educación contrastados con informes de organizaciones que evidencian problemáticas que han dificultado su alcance. Se expone una mirada proyectiva del derecho a la educación que no puede quedar encorsetado en la formulación de los nuevos Objetivos de Desarrollo Sostenible 2030. Habrá que ir más allá y avanzar en la verdadera democratización educativa.

Palabras clave: derecho a la educación, derechos humanos, Objetivos de Desarrollo del Milenio, democratización, política educativa.

\footnotetext{
Isabel Carrillo Flores es doctora en Pedagogía y posee estudios de Postgrado en Cooperación al Desarrollo. Profesora titular de la Facultad de Educación, Traducción y Ciencias Humanas de la UVic-UCC. Forma parte del Grupo de Investigación Educativa y del Centro de Estudios Interdisciplinares de Género. Ha sido directora del Departamento de Pedagogía y de la Cátedra Unesco Mujeres, Desarrollo y Culturas. Ha dirigido el Programa de Cooperación Educativa con Centroamérica. Contacto: Isabel.carrillo@uvic.cat
} 


\section{An evaluation of the millennium development goals and their impact on education}

\section{Abstract}

This article analyses the status of education as a fundamental right. The recent economic crisis and associated austerity policies have worsened the situation and, rather than diminish, educational inequalities have in fact increased. Firstly, the significance of education as a Human Right is examined. Secondly, the extent to which the MDGs have been met is reviewed, particularly with regard to education, where, as detailed in various reports produced by NGOs and by the United Nations, certain difficulties have impeded their being met. The Right to Education is examined in a projective way as a right that should not be limited to the vision contained in the new Sustainable Development Goals for 2030. It is necessary to go still further and advance towards a true democratisation of education.

Keywords: Right to education, human rights, Millennium development goals, democratisation, educational policy.

\section{Análise dos Objectivos de Desenvolvimento do Milénio e seu impacto sobre o direito à educaçáo}

\section{Resumo}

Este artigo discute a educação como um direito humano fundamental. O legado do s.XX mostra progressos, mas as políticas de crise e de ajustamento económico adotadas exacerbaram a desigualdade educacional. Neste contexto, as Metas de Desenvolvimento do Milênio projetadas para 2015 náo foram alcançados. Primeiro definiu a educação como um direito humano. Em segundo lugar objetivos são analisados, e os seus impactos são discutidos na educação, usando argumentos relatórios de organizaçôes que mostram problemas que impedem a sua realização. Finalmente, argumenta-se que o direito à educação não pode ser limitado na formulação das novas Objetivos de Desenvolvimento Sustentável 2030. Devemos avançar a verdadeira democratização educacional.

Palavras-chave: direito à educação, direitos humanos, Objectivos de Desenvolvimento do Milénio, democratização, política de educação. 


\section{LA EDUCACIÓN COMO DERECHO HUMANO}

En el transcurrir del siglo veinte la educación como derecho fundamental fue formando parte de las agendas políticas de las organizaciones internacionales y de los Estados. Ello fue posible gracias a los cambios políticos, económicos y sociales experimentados en diferentes países que, tras vivir graves conflictos negadores de la vida, avanzaron en la construcción de sociedades más democráticas, más conscientes de la dignidad de todas las personas y de la necesidad de crear las condiciones para la vivencia plena de los derechos humanos. Sin negar los esfuerzos realizados, el balance es desigual a nivel mundial y en el seno de los países.

El derecho a la educación sigue negándose a pesar de que en el año 2000, en el Foro Mundial celebrado en Dakar, 164 Gobiernos concertaron La Educación para Todos (EPT) (Unesco, 2000). El Informe de seguimiento de la EPT 2015 afirma que se ha avanzado en la escolarización en las etapas de la infancia y la adolescencia, así como en la paridad entre los sexos, especialmente en la enseńanza primaria (Unesco, 2015). Pero los resultados aún son discretos debido a problemas recurrentes en la historia. Se perpetúa un mundo dividido en un Norte enriquecido y un Sur empobrecido; para construir la paz los Estados activan guerras preventivas; las enfermedades y las hambrunas niegan la vida; los éxodos de población continúan provocando dolor, fracturas en las comunidades y ruptura de la cohesión social; se imponen modelos de desarrollo especulativo que inhiben el mismo; y las democracias no son una realidad a nivel mundial, como tampoco lo es la justicia social.

Los problemas son expresión de crisis vividas en todas las esferas, también en la educación, como puso de manifiesto Philip H. Coombs en su estudio de 1968 The World Educational Crisis: A Systems Analysis. Tras la Segunda Guerra Mundial la educación adquirió valor y pasó a ocupar un lugar central en las agendas políticas. Las expectativas se concentraban en el mensaje que la educación podía impulsar el desarrollo económico, y su democratización contribuiría a mejorar la calidad de vida a través de la eliminación de las injusticias. Se proyectaba un mundo de vivencia de los derechos humanos y la educación tenía que hacerlo posible. Coombs expresaba que estos deseos no se estaban cumpliendo debido a la existencia de una crisis de la educación a nivel global visible en que los cambios en la educación de los diferentes países no se habían dado con la misma intensidad, y los procesos de adaptación tampoco se habían producido a ritmos concordantes. Como consecuencia, las diferencias continuaban mostrando fracturas y desigualdades. La crisis sacaba a la luz las disparidades entre las aspiraciones educativas de la población, la falta 
de recursos y el aumento de los costes. Las propias inercias de los sistemas educativos y de las sociedades dificultan los cambios políticos y estructurales necesarios para garantizar la educación.

La educación como derecho humano se define como derecho moral y derecho jurídico (Rodríguez, 2011); un derecho universal, indivisible e interdependiente. Refiere a la dignidad de la persona: la dimensión personal relativa a la autonomía y la libertad; la dimensión social relativa a la no realización de acciones que niegan la integridad, y las garantías materiales que llevan a satisfacer las necesidades sin las cuales no se puede disfrutar de unas mínimas y aceptables condiciones de existencia (Ansuátegui, 2014). En esta orientación hay que significar el derecho a la educación como derecho fundamental sin el cual se niega la dignidad. Un derecho inmerso en un proceso dialéctico, de transformación y resignificación en una perspectiva inclusiva de todos los derechos. Un derecho que requiere de unos principios mínimos reconocidos por las Naciones Unidas que se tienen que expresar de forma simultánea y con la misma intensidad: educación asequible universal, gratuita y obligatoria; educación accesible sin restricciones, desde la primera infancia y a lo largo de la vida; educación aceptable, de calidad en los recursos materiales y en la formación docente; educación adaptable a la persona, atenta al principio de interés superior del alumnado (Tomasevski, 2004). En conjunto, una educación que en su expresión práctica debe fundamentarse en dos principios: la educabilidad y la educatividad. La primera refiere a educar sin exclusiones; remite al derecho a que las y los docentes confíen en que todas las personas pueden aprender y desarrollarse sin límites establecidos exteriormente. La segunda atañe a la educación como posibilidad y a la potencialidad de la enseńanza; exige considerar que el conocimiento es valioso, comunicable y puede tener carácter emancipador (Tenti, 2006). La ausencia de estos principios, y una baja o nula presencia de los indicadores señalados, vacía el contenido del derecho a la educación. Las consecuencias se observan en la vivencia de relaciones de exclusión.

Ante esta realidad, hay que pasar de las declaraciones de defensa de los Derechos Humanos como postulados del deber ser, a acciones políticas que junto a la dimensión axiológica y utópica de los derechos humanos, los contemple como un proyecto emancipatorio real y concreto (Perez Luño, 2006). Como derecho humano la educación debe vivirse como proyecto de emancipación que necesita de la convergencia de otros derechos, los que ya fueron normativizados en el pasado pero que deben ser revisados en los contextos actuales, y otros que surgen de nuevas necesidades. En este proceso habrá que tener presente que la tendencia a cuestionar la educación pública, 
a acusarla de los graves problemas de las sociedades del presente, conduce a una negación de la misma ignorando que es derecho humano consustancial a la persona, y que es universal e indivisible, porque los derechos humanos acompañan indefectiblemente a los seres humanos allí donde se encuentren y sean cuáles sean su condición y circunstancia (Vidal-Beneyto, 2006). La universalidad vinculada a la indivisibilidad se relaciona con la interdependencia, aunque son formas diferentes de relación entre los derechos. La primera refiere a la idea que todos los derechos constituyen un conjunto unitario y se encuentran interrelacionados, tienen que ser respetados y darse simultáneamente, puesto que todos están en la base de la democracia. La indivisibilidad se define como la forma más fuerte de relación, es bidireccional y constituye una relación en que un derecho es indispensable para otro y viceversa. Esta idea lleva a establecer que en los derechos humanos no hay derechos jerárquicamente superiores o prioritarios. Por su parte la interdependencia hace referencia a relaciones de apoyo entre los derechos; consiste en la contribución de un derecho para que se produzca el cumplimiento de otro (Añon, 2014). En esta perspectiva el derecho a la educación no es menos derecho y no puede estar marginalizado de las políticas. Al contrario, debe ser considerado en relación al conjunto de derechos humanos para que sea posible su vivencia plena.

\section{Impactos en la educación de los Objetivos de Desarrollo del Milenio}

Los estudios promovidos por las Naciones Unidas, junto a otros que realizan organizaciones no gubernamentales sobre la educación en el mundo, manifiestan que se han producido cambios substantivos, pero no suficientes, en la vivencia de la educación. A ello han contribuido las sucesivas crisis económicas que se han acompañado de contrarreformas en las políticas públicas cuyos impactos no se han circunscrito a un momento concreto, y no siempre han conllevado una mayor justicia social, más bien al contrario, pues se ha activado una progresiva minimización de los derechos fundamentales provocando un aumento de las desigualdades. Se ha justificado que dichas políticas son las únicas opciones posibles y las más óptimas, e inevitablemente tienen efectos colaterales. Como en las guerras, las decisiones políticas sobre otras esferas de la vida incorporan en sus paquetes de medidas a las víctimas colaterales que han sido seleccionadas a priori. La crisis se cierne en mujeres y hombres empobrecidos, y la pobreza es criminalizada. Es así que las personas pobres son candidatas naturales al daño colateral, porqué ya están marcadas de forma permanente con el doble estigma de la irrelevancia y la falta de mérito (Bauman, 2011). 
Además, los rasgos físicos y las identidades culturales, junto al sexo y la zona geográfica de origen o el territorio donde se vive, entre otros rasgos diferenciales, van a decidir si se está en posesión o no de un estatus de ciudadanía o no ciudadanía, de inclusión o exclusión, de formar parte de la centralidad o quedar en los márgenes de las periferias. Y la educación se convierte en un buen instrumento político para perpetuar tal situación. Una realidad denunciable que no puede ser objeto de ocultación ni de silencios por parte de las Naciones Unidas que deberían ser ejemplo de un hacer ético cuyo fundamento y fin sea la justicia social para dar respuesta a dos retos que no pueden desplazarse en el tiempo: impulsar y exigir la coherencia en las políticas sociales, entre ellas las educativas, actuando para eliminar las distancias existentes entre los discursos y las prácticas; y situar como centralidad prioritaria el derecho a la educación, actuando cuando este es marginalizado de las políticas de los Gobiernos.

En septiembre del 2000 la Asamblea General de las Naciones Unidas aprobó la Resolución 55/2 referente a la Declaración del Milenio, comprometiendo a los países firmantes a una alianza mundial para reducir los niveles de extrema pobreza. Con tal fin se establecieron ocho objetivos sujetos a plazo, los Objetivos de Desarrollo del Milenio (ODM). El año 2015 se estableció como clave para valorar la unidad de los países para poner fin a la pobreza y promover la prosperidad y el bienestar, requisitos necesarios para el derecho a la educación. Anualmente se han emitido informes de seguimiento que dan cuenta de los avances realizados, así como de los problemas detectados para su logro. El Informe 2015 pone de relieve que las personas más pobres son más vulnerables y sufren en mayor medida el desamparo, de modo que la agenda se ha tenido que reconsiderar y aplazar en el tiempo. Pero alargar en el tiempo no es suficiente. Se hace necesario revisar contenidos, finalidades y estrategias políticas pues, en sí mismos, los ODM plantean interrogantes éticos que ponen de manifiesto sesgos en una perspectiva dialéctica de los derechos humanos.

Los ODM priorizados hacen referencia a problemas mundiales que persisten, existiendo el peligro de naturalizarse y convertirse en rasgos endémicos de algunos países y grupos. De los ocho ODM el segundo se refiere a la educación, a lograr la enseńanza primaria universal, una finalidad restringida que no puede ser posible si al mismo tiempo no se alcanzan los restantes objetivos dado que se relacionan entre sí y no pueden observarse de forma aislada unos de otros. El derecho a la educación se niega en contextos de desigualdad, de pobreza, de enfermedad, de deterioro de la vida y de impedimentos al desarrollo humano, es decir, en contextos de fragilidad y no logro de los demás objetivos. 


\subsection{Empobrecimiento y precarización}

En su conjunto los ODM son expresión de pactos mínimos pero presentan limitaciones en su definición y en el compromiso político del deber de garantizarlos. El Objetivo 1 relativo a erradicar la pobreza y el hambre es limitado en su concreción de metas pues no aluden plenamente a la erradicación. Se estableció reducir a la mitad la proporción de personas con ingresos inferiores a 1,25 dólares al día; alcanzar el empleo pleno y productivo mediante un trabajo no precarizado; y reducir a la mitad la proporción de personas que padecen hambre. La pobreza es una realidad extendida y persistente, en parte como consecuencia de los modelos de desarrollo adoptados que han olvidado el desarrollo verdaderamente ético, humano y planetario, y lo es aquel que no perpetua la negación de una vida digna para todas las personas.

Las Naciones Unidas afirman que el objetivo de reducir a la mitad las tasas de pobreza extrema se ha alcanzado, sin embargo persiste, como también persiste la desigualdad en el empleo entre mujeres y hombres, así como el hambre, aunque sus focos contextuales pueden variar. Así lo pone de manifiesto Intermon Oxfam en sus informes de 2012 y 2014 que evidencian que la crisis económica mundial no ha frenado la concentración de riqueza de quienes más tienen, sino que ahonda más la desigualdad. Los datos concluyen que en el 2011 el 1\% más rico de la población mundial ganó la misma cantidad que el 56\% de población más pobre. En 2014 las 85 personas más ricas del planeta poseían la misma riqueza que la mitad más pobre de la humanidad. Las antiguas desigualdades, que en algunos países parecían en cierta forma superadas bajo el espejismo de la igualdad formal, se ven agravadas por el aumento de la desigualdad entre ricos y pobres.

También Cáritas en su Informe de 2014 muestra el camino ascendente de la pobreza en la realidad española, con el riesgo que el aumento de la desigualdad y la exclusión social se conviertan en crónicos. Los indicadores que lo ponen de manifiesto son: disminución del nivel medio de renta de las familias; menos empleo y más de carácter temporal; empobrecimiento creciente y derechos sociales menguantes. En el contexto de Europa el empobrecimiento afecta de forma especial a la niñez. Así lo recoge el Informe 2014 de Save The Children que sitúa en el centro la educación y la atención a la infancia como uno de los caminos para salir de la pobreza. Se pone de relieve que la infancia es un colectivo de una vulnerabilidad alta, no solo por su dependencia social y económica, sino también por el hecho de vivir en condiciones más pobres y más precarias. Sus vivencias educativas se empobrecen, pues aunque en muchos países la legislación reconoce la gratuidad de la enseñanza básica, 
esta no siempre incluye las actividades escolares complementarias planificadas en la concreción del currículum, ni los servicios escolares de comedor y transporte, como tampoco los libros y otros materiales que están sujetos a las cada vez más escasas ayudas compensatorias de las desigualdades.

Las políticas internacionales y nacionales deben partir de la constatación que no es posible conciliar la pobreza con el derecho a la educación, pues no pueden disfrutar de una educación plena quiénes han sido negados en su dignidad, han sido marginalizados, y destinados a vivir de forma precarizada.

\subsection{El estigma del género}

La realidad descrita no afecta de forma igual a mujeres y hombres. El Objetivo 3 hace referencia a promover la igualdad de género y empoderamiento de la mujer, y se concreta en la meta de eliminar las desigualdades de género en la enseñanza. Tal meta alude directamente a la educación que debería tener como finalidad erradicar el legado histórico del androcentrismo. Todas las políticas deben contemplar la transversalidad del género, pues la igualdad en la diferencia no puede ser discutible, como no lo es la dignidad de la persona. En este sentido habrá que evaluar el impacto de género de los ODM dado que las problemáticas que abordan afectan a lo largo de la vida de forma diferente a mujeres y hombres. Es en el momento de nacer — nacer nińa, nacer niño - cuando se establece la diferencia sexual como rasgo distintivo que se proyectará impregnando las relaciones de cada ser humano con la realidad (Rivera Garreta, 2005). Se hace necesario atender al género —asociado al sexo- como indicador de análisis para evaluar los cambios, para observar la mejora de la vida de las personas atendiendo al hecho que los efectos de un programa político no son neutros, pues mujeres y hombres pueden no estar en igualdad de condiciones (Murguialday, Vázquez, González, 2008).

Históricamente no solo los problemas han sido, y son vividos en el presente, de forma más generalizada e intensa por las mujeres, sino también por todas aquellas personas que están marginalizadas de una realidad normativizada en dos — dos sexos, dos géneros- Existen resistencias a considerar la diferencia más allá de la norma establecida. Mucho más en aquellos países en que la legislación perpetúa la situación de subordinación y opresión de las mujeres — y de los demás grupos no sujetos a norma-, siendo la educación un instrumento para mantener tal realidad, ya sea porque se niega o limita el acceso, ya sea porque se activan mecanismos de segregación a través de centros diferenciados y/o currículums también diferenciados por sexos; o centros mixtos con una cultura escolar que tiene como centralidad lo masculino, dado que se establece 
el androcentrismo como valor; o centros que declaran la coeducación y en su hacer continua operando el currículum oculto que mantiene los instrumentos de reproducción sexista. No obstante, aunque la educación es una vía de reproducción social y cultural, también lo puede ser de transformación. Pero no es lo que está ocurriendo, o no de forma suficiente. El Informe 2014 de Unicef sobre el estado mundial de la infancia pone de relieve que no se ha hecho efectivo el derecho a vivir y desarrollarse, pues continúan las muertes de menores de 5 años por causas prevenibles; además niñas y niños realizan trabajos que menoscaban su derecho a la protección contra la explotación económica e infringen su derecho a aprender y jugar, y sufren violencias degradantes tanto en sus hogares como en las escuelas. La situación para las niñas es más alarmante.

La igualdad no es posible sin el valor de la diferencia, ella es su razón de ser. El problema surge cuando la diferencia es argumento para la desigualdad; cuando es utilizada como factor de desvalor que actúa como indicador que mide quién merece o no merece un bien. El sexo ha sido y es estigma que señala y fuerza para determinar el grupo que debe ser marginalizado de la educación, y con ello de la vida más allá de los muros reales y simbólicos que encierran en el ámbito privado y no permiten habitar el mundo público.

El derecho a la educación no va a ser posible sin un cambio en los sistemas y modelos educativos con el fin de orientar sus principios y sus finalidades hacia la deconstrucción de los géneros femenino y masculino que trazan trayectorias de desigualdad.

\subsection{Salud débil y entornos no sostenibles}

Las cuestiones planteadas deben ser consideradas en relación a los restantes ODM. Respecto al Objetivo 4 sobre reducir la mortalidad infantil en menores de 5 años, es necesario señalar que no es éticamente aceptable acuerdos que siguen admitiendo la perpetuación de la muerte de niñas y de niños que no van a tener opción a la vida, siendo especialmente grave en países que silencian y perpetúan el infanticidio de las niñas y los abortos selectivos. También son demasiado laxos el Objetivo 5 sobre mejorar la salud materna, y el 6 relativo a combatir el VIH/SIDA, la malaria y otras enfermedades. Se declara que hay que mejorar la salud de las mujeres y de las nińas en todas las etapas de su vida, y se insiste en el hecho que los estudios muestran que cuando la salud de las madres mejora, también mejora la de sus hijas e hijos, metas positivas dado que la salud es requisito necesario para vivir la educación. Sin embargo la propia Organización Mundial de la Salud indica que las políticas en salud pública ignoran los impactos de género, y como consecuencia 
algunas enfermedades de las mujeres no están reconocidas ni contempladas en los presupuestos públicos, son marginales. La tendencia a la privatización de los derechos sociales alerta de lo preocupante de la situación, pues la salud es también un derecho humano y no puede ser objeto de negocio. No puede dejarse en manos de las empresas la decisión sobre qué servicios ofrecer, y a quién, así como el coste de los mismos. Como tampoco puede dejarse a la iniciativa privada decidir qué investigaciones realizar en función de los beneficios económicos que se esperan obtener, sin tener en cuenta que la finalidad debe ser el procurar el bienestar de todas las personas sin exclusiones. No es ético externalizar lo que es deber de garantizar que la salud no sea derecho solo para unos grupos, excluyendo a los etiquetados como no dignos ni merecedores de la misma. El derecho a la salud y el derecho a la educación están íntimamente relacionados. Sin salud no puede vivirse la educación, y sin esta una vida para la salud y el bienestar tampoco es posible.

Añadir que es condición para el logro de los objetivos indicados lo dispuesto en el Objetivo 7, garantizar la sostenibilidad del medio ambiente, y el Objetivo 8, fomentar una alianza mundial para el desarrollo, objetivos que deberían considerar las aportaciones sobre otros modelos de desarrollo. Ejemplo es el eco-feminismo que propone una democracia de la tierra basada en economías vivas y en democracias económicas. Para ello se precisan de políticas que fomenten sistemas sostenibles, diversos y plurales que tengan por objeto proteger a la naturaleza y a las personas para que estas, en sus contextos de vida próximos, puedan elegir sus formas de vida atendiendo al bien común (Shiva, 2006). En esta perspectiva los Estados deben ser garantes de los derechos humanos como mínimos necesarios para el desarrollo humano sostenible. Ello exige alejarse de los parámetros de desarrollo del capitalismo neoliberal recuperando aportaciones como las de Amartya Sen que en 1990 definió, en el Programa de las Naciones Unidas para el Desarrollo, el índice de desarrollo humano (IDH) que relaciona el desarrollo social y económico: el ingreso nacional bruto por cápita que refiere a la dimensión estándares de vida; la esperanza de vida al nacer relacionada con la dimensión salud; el promedio de años de escolaridad y los años esperados, los índices de matrícula y de alfabetización, relativos a la dimensión educación.

El balance de los ODM plantea dudas sobre si las políticas internacionales y nacionales se están orientando hacia un verdadero desarrollo humano. Surge la duda porque la realidad no deja de mostrarnos cómo en los modelos de sociedad que se priorizan se ensalzan los valores reactivos de cortoplacismo, competitividad e individualismo que lo inhiben (Cortina, 2010). Con ello la educación es insistentemente negada. 


\section{Proyectar la educación como derecho fundamental}

Como los ODM comentados, puede sorprender lo limitado del Objetivo 2 que hace referencia a lograr la enseńanza primaria universal, objetivo que se concretó en la meta de asegurar que las nińas y los nińos de todo el mundo pudieran terminar un ciclo completo de enseñanza primaria. Sin embargo el objetivo no parece contradecir el artículo 26 de la Declaración Universal de los derechos humanos que establece que toda persona tiene derecho a la educación y que esta debe ser gratuita, al menos en lo concerniente a la instrucción elemental y fundamental que será obligatoria. Este ODM pone de manifiesto que desde 1948 el derecho a la educación sigue siendo un reto pendiente, como pendiente es adoptar una perspectiva generacional y dinámica de los derechos que permita resignificarlos y generar otros nuevos de acuerdo a las necesidades de las sociedades del presente. En este sentido la educación, como derecho humano, no puede quedar reducida a la enseñanza primaria, pues aun siendo posible y sin desmerecer sus impactos en el desarrollo humano, no va a ser suficiente para erradicar las desigualdades. La división jerarquizada entre los países y microrrealidades llamadas del Norte —enriquecidas - y los países y microrrealidades llamadas del Sur —empobrecidas — seguirá existiendo al amparo de itinerarios diferentes: un itinerario para aquellas personas cuyo horizonte se queda limitado a la enseñanza primaria, única etapa que se establece como obligatoria; y otro itinerario para aquellas otras personas cuyo horizonte no tiene límites, sino que gozan de un amplio abanico de opciones pre y postobligatorias orientadas al éxito, a ocupar los espacios de decisión que afectan no solo a sus vidas, sino a las vidas de quiénes están privados de participar y decidir. El derecho a la educación no admite los monopolios del conocimiento, porque este es bien público imprescindible para el bienestar sin exclusiones. Por ello es exigible a los Gobiernos el no perpetuar y erradicar los circuitos educativos diferenciados, el de una escuela precaria sin condiciones mínimas para pobres, y una escuela bien dotada de más y mejores condiciones para ricos (Gentili, 2011).

Si alcanzar el objetivo de la enseñanza primaria universal es urgente, también lo es el reconocer que aunque necesario es limitado en su proyección. Unicef informa que se ha demostrado que proveer a niñas y niños de una educación básica de calidad podría impulsar el crecimiento económico anual en un $2 \%$ en los países de bajos ingresos, y además sería posible librar de la pobreza al $12 \%$ de las personas pobres si la totalidad de estudiantes de los países pobres tuvieran aptitudes de lectura básicas. Por otra parte se ha evidenciado que el incremento mundial que ha experimentado la educación de las mujeres 
ha evitado más de cuatro millones de muertes infantiles, y que cada año adicional de escolarización puede propiciar un aumento de los ingresos de la mujer de entre el $10 \%$ y el $20 \%$. Todo ello sin obviar que 1 millón de dólares invertidos en educación y aptitudes equivale a 10 millones de crecimiento económico. Estos son datos nada despreciables, y lo son porque un contenido restrictivo de los objetivos puede llevar a justificar políticas restrictivas en derechos sociales, no solo utilizando como excusa las crisis, sino también las propias directrices de los organismos internacionales que marcan las líneas y fines prioritarios en educación. El Informe de 2015 sobre los ODM muestra los avances en el Objetivo 2, como es el hecho que a nivel mundial la tasa neta de matriculación en la enseńanza primaria se ha ampliado al mismo tiempo que se ha reducido a la mitad el número de nińas y niños que no asiste a la escuela; son más los que completan la escuela primara en los países con menos ingresos; y es mayor la tasa de alfabetización de jóvenes.

Avances parciales, pues continúan las disparidades que ponen en peligro la educación desde la primera infancia, no solo en países donde la educación no era, sino también en contextos donde el derecho a la educación había ido generalizándose. El Informe 2014 de Unicef sobre la crisis en países ricos indica que allí donde la educación había experimentado avances notablemente significativos, el empobrecimiento está destruyendo de forma acelerada tal realidad y la niñez es el grupo social más perjudicado: 76,5 millones de niñas y niños viven por debajo del umbral de la pobreza de sus respectivos países (2,6 millones más que en 2008). Como se ha seńalado la persistencia de la pobreza condiciona el alcance de los ODM, actúa como inhibidor del bienestar de las personas, y aparta a las niñas y a los niños de la vivencia plena de la educación, porque en sus vidas precarizadas la educación como derecho les está negada.

La educación sigue siendo reto pendiente y continua estando presente en la Agenda Post-2015 sobre los 17 Objetivos de Desarrollo Sostenible (ODS) para el 2030. El Objetivo 4 establece garantizar una educación inclusiva, equitativa y de calidad, así como promover oportunidades de aprendizaje durante toda la vida. Se plantea una enseñanza primaria y secundaria completa y gratuita que genere aprendizajes, así como reducir el analfabetismo de las personas adultas para acceder a un empleo óptimo. En los años venideros habrá que analizar críticamente los significados teóricos y prácticos que adoptan tales formulaciones, y habrá que hacerlo para que las políticas que se desarrollen sean un verdadero contrapunto a la tendencia privatizadora de la educación, como ya puso de manifiesto las Naciones Unidas en el Informe de 2004 presentado por Katarina Tomasevski, Relatora Especial sobre el Derecho a la Educación, quien denunció que el derecho se ha sustituido por el acceso a la educación, 
y la obligación de los Gobiernos de velar para que al menos la enseñanza obligatoria sea gratuita se ha atenuado colocando dicha palabra entre comillas — «gratuita»—. Para la autora del informe estas variaciones lingüísticas tienen por objeto destacar que se debe financiar la educación, negando implícitamente que su financiación deba hacerse con cargo a recursos públicos.

En un contexto de expansión del capitalismos del desastre, de eliminación de la esfera pública y de un gasto social esquelético (Klein, 2007), los Gobiernos aprueban leyes que conciben la educación desde una perspectiva mercantilista, más que como un derecho fundamental universal. La educación se presenta como dependiente de los procesos económicos, del sistema productivo, anteponiendo las necesidades de las empresas liberalizadas, es decir, se instrumentaliza y se pone al servicio de intereses sectoriales. Las instituciones educativas se privatizan y se convierten en empresas que compiten entre ellas, y se promueve que los centros se vuelvan más flexibles con la finalidad de adaptarse con mayor rapidez a los rápidos cambios del mercado laboral. La educación pasa a ser un bien de consumo que se compra y se vende o, como se ha apuntado, un producto que solo podrá estar disponible en el mercado en función de la productividad y las ganancias económicas, y solo podrá tenerse si se dispone del suficiente poder adquisitivo (Jares, 2005 y Hirtt, 2003). Sin duda ello tiene consecuencias en determinados grupos de población que van siendo negados, como ciudadanas y ciudadanos, y marginados de la sociedad de la que forman parte. En su estatus estigmatizado las personas excluidas son desposeídas de todo derecho humano y, por tanto, de la educación como derecho porque no pueden pagarla.

\subsection{El deber de procurar el derecho a la educación}

El camino que se ha trazado en este texto no es neutral, como tampoco lo es la educación ni las políticas que la definen y proyectan. En conjunto se ha querido poner de relieve que si bien las Naciones Unidas han adoptado una posición beligerante respecto al reconocimiento de la educación como derecho humano, sus declaraciones, objetivos e informes plantean interrogantes éticos y resultan insuficientes para cambiar una realidad que reproduce las desigualdades educativas. En el presente es urgente un posicionamiento claro de exigencia para reconocer el valor de la educación y pensarla como derecho que no puede ser negado. En el aquí y ahora no sirve el realizar promesas de futuro y agendas para tiempos diferidos, pues aplazar en el tiempo comporta una cierta ocultación de un reto pendiente: el deber de garantizar la educación como derecho fundamental. 
Si bien los nuevos ODS para 2030 pueden ser necesarios por cuanto visibilizan los problemas y proyectan su erradicación, no es en el mañana sino desde el hoy que es imprescindible impulsar políticas orientadas a dar respuestas justas desde un enfoque holista y sistémico. Es en esta perspectiva que se indican acciones mínimas que, sin ser las únicas posibles, pueden movilizar un hacer políticamente ético ante la educación negada.

Primera. La responsabilidad de desvelar lo oculto con la finalidad de mostrar que los principios y las intenciones de las políticas educativas no son neutrales. Esto significa visualizar las ideologías que han acompañado la educación, no negar la memoria de los hechos y reconocer las dificultades del derecho a la educación, en su trayectoria histórica y en el presente, siendo conscientes que no todo está dado y que aún quedan muchas barreras. Ello es necesario para proyectar el futuro en un equilibrio justo de utopía y posibilidad, con el convencimiento que la educación no está determinada ni puede determinar a las personas y sus vidas.

Segunda. Defensa de una educación pública orientada a la corrección de la tendencia ascendente de la privatización de la educación. La educación no puede mercantilizarse, como derecho humano fundamental no es un producto más de consumo que se compra y se vende, o un producto que cotiza en bolsa y que en función de la oferta y la demanda aumenta su valor o se devalúa. Las reglas del mercado no son para la educación. Los derechos son consustanciales a las personas, y los Estados y sus Gobiernos no pueden ser cómplices de intereses empresariales ajenos al bienestar de la ciudadanía. Al contrario, tienen la responsabilidad política de ser garantes del derecho a la educación.

Tercera. Actuar desde y para la equidad desde un posicionamiento claro y sin concesiones de la educación sin exclusiones, lo que significa que es una educación que se fundamenta en el principio ético de justicia social porque su finalidad es la igualdad en la diferencia. El compromiso individual y colectivo es el de actuar de forma justa a través de prácticas que sean expresión de la igualdad de derechos y oportunidades, al mismo tiempo que se actúa exigiendo políticas de equidad frente a las desigualdades.

Cuarta. Construir la ciudadanía democrática a través de una educación que enseña a aprender a vivir humanamente aprendiendo a dar respuestas éticas a los dilemas que se viven en el mundo. Es esta una educación que necesita de la democracia para ser y proyectar acciones que empoderan y son liberadoras, que forma en la ciudadanía del compromiso y del cuidado, de participaciones dialógicas, reconocimientos y solidaridades.

Quinta. Las agendas politicas han de ser para el presente, hay que movilizarse y romper con la parálisis del no hacer. Es urgente no dejarse arrastrar por la 
confusión con la seguridad que la educación del hoy se niega a ser negada, con la esperanza que alienta y guía el aprender a aprender a construir el derecho a la educación, sabiendo que la educación es compromiso con el mundo y es gesto de amor por la humanidad. Hay que hacer desde un claro posicionamiento político para la vida ética que se quiere vivir, aunque ello sea en tiempos de aires difíciles y exija el esfuerzo de remar a contracorriente.

\section{REFERENCIAS BIBLIOGRÁFICAS}

Ansuátegui, F.J. (2014). Los derechos sociales en tiempos de crisis. Algunas cuestiones sobre su fundamentación. En Bernuz, M.J., Calvo, M. (Ed.). La eficacia de los derechos sociales (pp. 23-42). València: Tirant lo Blanch.

Añon, M.J. (2014). Derechos humanos y obligaciones positivas. En Bernuz, M.J., Calvo, M. (Ed.). La eficacia de los derechos sociales (pp. 43-71). Valencia: Tirant lo Blanch.

Bauman, Z. (2011). Daños colaterales. Desigualdades sociales en la era global. Madrid: Fondo de Cultura Económica de España.

Cáritas (2014). Precariedad y cohesión social. Recuperado de: http://www.solidarios.org.es/wp-content/uploads/foessa-precariedad-cohesion-social.pdf

Coombs, P.H. (1968). The World Educational Crisis: A Systems Analysis. Oxford University Press.

Cortina, A. (2010). Los valores de una ciudadanía activa. En B. Toro, A. Tallone (Coords.). Educación, valores y ciudadania (pp. 95-108). Madrid: OEI.

Gentili, P. (2011). Pedagogía de la igualdad. Ensayos contra la educación excluyente. Buenos Aires: Siglo XXI-CLACSO.

Jares, X. (2005). Educar para la verdad y la esperanza. En tiempos de globalización, guerra preventiva y terrorismos. Madrid: Ed. Popular.

Hirtt, N. (2003). Los nuevos amos de la escuela. El negocio de la enseñanza. Madrid: Minor Network.

Intermon Oxfam (2012). Crisis, desigualdad y pobreza. Recuperado de: http:// www.oxfamintermon.org/sites/default/files/documentos/files/Informe_ IO_Crisis_desigualdad_y_pobreza_300113.pdf

Intermon Oxfam (2014). Iguales. Acabemos con la desigualdad extrema. Es hora de cambiar las reglas. Recuperado de: http://www.oxfamintermon.org/ sites/default/files/documentos/files/InformeIGUALES_AcabemosConla DesigualdadExtrema.pdf

Klein, N. (2007). La doctrina del xoc. L’ascens del capitalisme del desastre. Barcelona: Empúries. 
Murguialday, C., Vázquez, N., González, L. (2008). Un paso más: evaluación del impacto de género. Barcelona: Cooperacció.

Naciones Unidas (2000). Resolución 55/2 Declaración del Milenio. Recuperado de: http://www.un.org/spanish/milenio/ares552.pdf

Naciones Unidas (2004). Los derechos económicos, sociales y culturales: el derecho a la educación. Informe presentado por Katarina Tomasevski, Relatora sobre el derecho a la educación. Resumen. Recuperado de: http://www.oei.es/ decada/portadas/G0410331.pdf

Naciones Unidas (2015). Objetivos de desarrollo del milenio. Informe 2015. Es hora de la acción mundial, por las personas y el planeta. Recuperado de: http:// www.un.org/es/millenniumgoals/pdf/2015/mdg-report-2015_spanish.pdf

Naciones Unidas. Declaración Universal de Derechos Humanos. Recuperado de: http://www.un.org/es/documents/udhr/

Pérez Luño, A.E. (2006). La tercera generación de derechos humanos. Navarra: Aranzadi.

PNUD. Informes sobre desarrollo humano. Recuperado de: http://www.undp.org/ content/undp/es/home/librarypage/hdr.html

PNUD. Objetivos de Desarrollo Sostenible. Recuperado de: http://www.undp.org/ content/undp/es/home/mdgoverview/post-2015-development-agenda. html

Rivera Garreta, M.M. (2005). La diferencia sexual en la historia. Valencia: PUV.

Rodríguez, M.E. (2011). Claves para entender los nuevos derechos humanos. Madrid: Los libros de la catarata.

Save The Children (2014). Pobreza infantil y exclusión social en Europa. Una cuestión de derechos. Recuperado de: https:/www.savethechildren.es/sites/ default/files/imce/docs/europa_pobreza_infantil_y_exclusion_social_en_ europa.pdf

Shiva, V. (2006). Manifiesto para una democracia de la tierra. Barcelona: Paidós.

Tenti, E. (2006). El oficio docente. Vocación, trabajo y profesión. Buenos Aires: Siglo XXI.

Tomasevski, K. (2004). El asalto a la educación. Barcelona: Intermon Oxfam.

Unesco (2000). Marco de acción de Dakar. Educación para todos: cumplir nuestros compromisos comunes. Recuperado de: http://unesdoc.unesco.org/ images/0012/001211/121147s.pdf

Unesco (2015). La educación para todos 2000-2015. Logros y desafios. Recuperado de: http://unesdoc.unesco.org/images/0023/002324/232435s.pdf

Unicef. Educación para todos los niños. Recuperado de: http://www.unicef.es/ infancia/educacion-para-todos-los-ninos 
Unicef (2014). Los niños de la recesión. El impacto de la crisis en el bienestar infantil en los paises ricos. Recuperado de: http://www.unicef.org/ecuador/ Losninosdelarecesion.pdf

Vidal-Beneyto, J. (Ed.) (2006). Derechos humanos y diversidad cultural. Globalización de las culturas y derechos humanos. Barcelona: Icária. 